Journal of Applied Veterinary Sciences, 5 (2): 13-24 (2020).

ISSN: Online: 2090-3308, Print: 1687-4072

Journal homepage : https://javs.journals.ekb.eg

\title{
Evaluation of Sensory and Bacteriological Quality of Meatball Supplemented by Spirulina platensis
}

\author{
Kareman S. Awadalla', Ali M. Ahmed ${ }^{1}$,Mohamed M. Abdel-Daim², Nagwa T. Elshraway ${ }^{3}$, \\ Mariam A. Abdel-Wahab ${ }^{4^{*}}$ \\ ${ }^{1}$ Food Hygiene Department, Faculty of Veterinary Medicine, Suez Canal University, Ismailia, Egypt. \\ ${ }^{2}$ Pharmacology Department, Faculty of Veterinary Medicine, Suez Canal University, Ismailia, Egypt. \\ ${ }^{3}$ Food Hygiene Department, Faculty of Veterinary Medicine, New Valley University, Egypt. \\ ${ }^{3}$ University of Jeddah, College of Science, Department of Biology, Saudi Arabia \\ ${ }^{4}$ Dept. of Food Safety and Technology, Faculty of Veterinary Medicine, Minia University, Egypt \\ *Corresponding authors, Mariam A. Abdel-Wahab E-mail: dr_mariam79@yahoo.com
}

\begin{abstract}
Spirulina "Superfood" is the common name of blue-green microalgae, which have a spiral cellular structure belonging to two genera Spirulina and Arthrospira which consist of (55-70\% dry weight) protein, (5-6\%) lipid, carbohydrates, vitamins, minerals and pigments. According to FDA Spirulina is approved and safe to be employed as a food additive. Ten $\mathrm{kg}$ of fresh minced meat was purchased from different retail markets within Ismailia province, mixed thoroughly with common salt and divided into seven portions; control, $0.5 \%, 1 \%, 1.5 \%, 2 \%, 3 \%$ and 5\% concentrations of added Spirulina platensis powder. About 500g from control and treated samples were formed into small meatballs, refrigerated at $4^{\circ} \mathrm{C}$ and examined for sensory and bacteriological evaluation at (zero, 2, 3, 4, 6, 8 and 9 days) of storage. The results of this study showed that the addition of Spirulina platensis had an adverse effect on the colour of both raw and cooked meatball samples, but gave an acceptable smell. Consistency of raw meatball samples was acceptable at different concentrations of Spirulina platensis, while it was mildly affected in cooked meatball samples especially at $5 \%$ concentration. The Taste of cooked meatball samples was not affected by the addition of different concentrations of Spirulina platensis. The obtained results revealed that the addition Spirulina platensis has the ability to reduce the growth of total aerobic bacteria, Enterobacteriaceae spp. and Staphylococcus aureus.
\end{abstract}

Keywords: Enterobacteriaceae species, food processing, Meatball,

Original Article:

DOI:HTTPS://DX.DOI.ORG/10.2 1608/JAVS.2020.85554

Received:05 Jan., 2020.

Accepted : 19 Feb., 2020.

Published in April 2020.

This is an open access article under the term of the Creative Commons Attribution $\quad 4.0 \quad$ (CC-BY) International License . To view a copy of this license, visit http://creativecommons.org/licenses/ by/4.0/

J. Appl. Vet Sci., 5(2 ):13-24. Spirulina platensis, Staphylococcus aureus.

\section{INTRODUCTION}

Spirulina is the common name of blue-green microalgae, which have a spiral cellular structure belonging to two genera Spirulina and Arthrospira. Spirulina known as "Superfood" due to its composition which consists of $(55-70 \%$ dry weight) protein including amino acids such as (Valine, Isoleucine, tryptophan, phenylalanine, threonine, methionine, lysine and leucine), (5-6\%) lipid mainly composed of omega-6, omega-3, gamma-linolenic acid (36\% total PUFAs), (glycolipids, sulfolipids), carbohydrates (mannose, Glucose, galactose, xylose and rhamnose), carotenoid $(4000 \mathrm{mg} / \mathrm{kg}),(1.5-2 \%)$ vitamins (A, B, E), minerals (iron, calcium, manganese, zinc, magnesium, selenium and potassium) and pigments (chlorophyll, carotenoids, xanthophylls) and blue pigment which known as phycocyanin (Balch and Balch, 2000; McCarty, 2007; Hosseini, et al., 2013; Ghaeni and Roomiani, 2016 and Pyne, et al., 2017). According to the Food and Drug Association (FDA and The National Health Surveillance Agency (ANVISA) Spirulina is approved to be safely employed in meat science and medicine as a food additive without toxicological effects. Among many Spirulina species S. platensis, $S$. maxima and $S$. fusiformis are considered the most important spp. due to their high nutritional value and therapeutic potential (Khan, et al., 2005; Soheili, and Khosravi-Darani, 2011 and Navacchi et al., 2012).

Spirulina platensis extract was found to have many biological activities such as antimicrobial (antibacterial, antiviral, antifungal), antiprotozoal and anticancer (Usharani, et al., 2012). Spirulina platensis 
has an antimicrobial effect against a wide range of micro-organisms including gram-positive bacteria such as Staphylococcus aureus, Streptococcus thermophiles and Lactococcus spp., gram-negative bacteria such as Enterobacteriaceae spp., and Escherichia coli and fungi such as Aspergillus niger and Candida albicans (Mendiola et al., 2007 and Mala et al., 2009). Meat and its products are regarded as one of the highest nutritive value food (FAO, 2015). Meatball is one of the meat products defined as round meat prepared from minced meat (cattle, goat, duck, chicken) that mixed with some additives to improve its acceptability and safety.

Modern lifestyle enhances the demands for ready-to-eat food which is known as "convenience foods" which is very popular globally. The meatball is one of these; "convenience foods" that prepared from a mixture of $53 \%$ ground lean beef, $17 \%$ fat, and some salts (monosodium glutamate (MSG), phosphates, sodium chloride) then formed to the desired shape and frozen until cooking (Purnomo and Rahardiyan, 2008). Extending the shelf-life of different types of food, especially meat and meat product is the main aim in the food industry. Microbial spoilage is the main hazard which decreases the meat shelf life. Preservation of meat and meat products aimed mainly to reduce and/or inhibit the growth of different microorganisms safely to extend the shelf life of it. (Yilmaz and Demirci, 2010).

Recently, enhancement of food safety has been the major interest by increasing usage of natural preservatives which has antioxidant and antimicrobial properties of their essential oils. At the same time, there is an increased preference for natural food and food ingredients, which are generally believed to be safer, more healthy and less subject to hazards than that containing artificial additives especially in meat products which are highly susceptible to microbial growth that can cause spoilage and contributes to foodborne diseases in human (Demule et al., 1996, Xue, et al.,2002 and Abd El-Malek, 2017).

Although scientists concerned the nutritive and antioxidant values of Spirulina platensis with few concerns on its antimicrobial activity and nearly none of them use these antimicrobial characters as natural preservatives in food. The objectives of this study were to evaluate the organoleptic properties and microbial load of meatball after addition of Spirulina platensis with different concentrations and to determine the most acceptable level of Spirulina platensis.

\section{MATERIALS AND METHODS}

\section{Collection and Preparation of Samples}

A total of ten kilograms of minced beef meat was purchased from different retail markets within Ismailia province. Samples were collected and transferred under sterile conditions without delay in pre-cooled insulated containers with ice packs to the laboratory. Spirulina platensis powder was obtained from; Spirulina capsules (GNC earth genius Spirulina, General Nutrition Corporation, Pittsburgh, PA 15222, USA). Fresh minced beef was mixed thoroughly with common salt and divided into seven portions, each weighted $500 \mathrm{gm}$. First portion was used as a control, while the other portions were mixed with powder of Spirulina platensis in different concentrations $(0.5 \%$, $1 \%, 1.5 \%, 2 \%, 3 \%, 5 \%$ ), then stored on the refrigerator shelf at $4^{\circ} \mathrm{C}$, and examined periodically for sensory and bacteriological evaluation at zero, 2 days, 3 days, 4 days, 6 days, 8 days and 9 days of storage.

\section{Sensory evaluation}

About 500g from each treated sample was formed into small meatballs. After that, the samples were divided into two parts (raw \& cooked) and tested by the panel members $(n=10)$ who were not trained in the sensory evaluation of meat. Five characteristic points were given to panellists according to the following score: 1- very bad, 2- bad, 3- good, 4- very good, and 5- excellent. It was required from the panellists to consider the aforementioned points for evaluation of colour, flavour and consistency of the meatball in addition to taste in cooked samples.

\section{Bacteriological evaluation}

Twenty-five grams from each sample were aseptically taken, diluted with $225 \mathrm{ml}$ of sterile $0.1 \%$ peptone water $(w / v)$ in a sterile stomacher bag and homogenized in a stomacher (LAB-BLENDER, 400) for one minute, then the mixture was allowed to settle, for 5 minutes at room temperature. One $\mathrm{ml}$ of the homogeneous sample was transferred aseptically to a test tube containing $9 \mathrm{ml}$ sterile $0.1 \%$ peptone water $(w / v)$ from which further tenfold decimal serial dilution up to $10^{-8}$ were prepared. The bacteriological investigations were performed from the prepared samples by using Plate Count Agar for determination of total aerobic count (ICMSF, 1996), Mannitol salt agar for total Staphylococcal aureus count (ICMSF, 1996) and Violet Red Bile Glucose Agar for Enterobacteriaceae count (ISO, 2004). Brain heart infusion (BHI) broth was used for the identification of Staph aureus isolates (ICMSF, 1996).

\section{Statistical Analysis}

The statistical program, GraphPad Instant version 5 for windows 7 , was used for determination of means, the analysis of variance between the different data and treatment in this study were determined using standard error and analysis of variance $(P<0.05)$. The figures were used to display the significant difference between the obtained results in this study(GraphPad Instant, 2009). 
Kareman S. Awadalla et al......

\section{RESULTS}

Table 1: Effect of addition of different concentrations of Spirulina platensis on sensory quality of raw meatball samples

\begin{tabular}{|c|c|c|c|c|c|c|c|c|c|c|c|c|c|c|c|}
\hline \multirow{2}{*}{ Grade } & \multicolumn{5}{|c|}{ Colour } & \multicolumn{5}{|c|}{ Smell } & \multicolumn{5}{|c|}{ Consistency } \\
\hline & 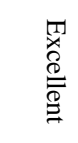 & 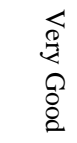 & $\begin{array}{l}0 \\
\stackrel{8}{0}\end{array}$ & $\begin{array}{l}\mathscr{0} \\
\ddot{0}\end{array}$ & 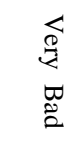 & 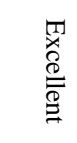 & 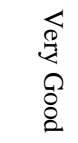 & $\begin{array}{l}0 \\
\varnothing \\
\varnothing\end{array}$ & 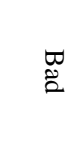 & 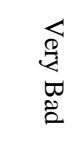 & 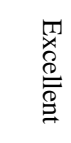 & 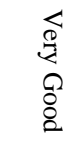 & $\begin{array}{l}0 \\
8 \\
8\end{array}$ & $\begin{array}{l}\mathscr{J} \\
\ddot{0}\end{array}$ & 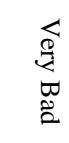 \\
\hline Control & $30 \%$ & $30 \%$ & $40 \%$ & $00 \%$ & $00 \%$ & $20 \%$ & $60 \%$ & $10 \%$ & $10 \%$ & $00 \%$ & $20 \%$ & $50 \%$ & $30 \%$ & $00 \%$ & $00 \%$ \\
\hline $0.5 \%$ & $00 \%$ & $10 \%$ & $10 \%$ & $70 \%$ & $10 \%$ & $10 \%$ & $10 \%$ & $40 \%$ & $30 \%$ & $10 \%$ & $00 \%$ & $40 \%$ & $60 \%$ & $00 \%$ & $00 \%$ \\
\hline $1 \%$ & $00 \%$ & $10 \%$ & $10 \%$ & $30 \%$ & $50 \%$ & $00 \%$ & $30 \%$ & $10 \%$ & $30 \%$ & $10 \%$ & $00 \%$ & $50 \%$ & $20 \%$ & $20 \%$ & $10 \%$ \\
\hline $2 \%$ & $00 \%$ & $10 \%$ & $10 \%$ & $30 \%$ & $50 \%$ & $20 \%$ & $30 \%$ & $10 \%$ & $30 \%$ & $10 \%$ & $10 \%$ & $30 \%$ & $50 \%$ & $10 \%$ & $00 \%$ \\
\hline $3 \%$ & $00 \%$ & $00 \%$ & $10 \%$ & $30 \%$ & $60 \%$ & $00 \%$ & $30 \%$ & $30 \%$ & $40 \%$ & $00 \%$ & $10 \%$ & $20 \%$ & $60 \%$ & $10 \%$ & $00 \%$ \\
\hline $5 \%$ & $00 \%$ & $00 \%$ & $10 \%$ & $20 \%$ & $70 \%$ & $00 \%$ & $30 \%$ & $40 \%$ & $30 \%$ & $00 \%$ & $30 \%$ & $30 \%$ & $40 \%$ & $00 \%$ & $00 \%$ \\
\hline
\end{tabular}

Table 2: Effect of addition of different concentrations of Spirulina platensis on sensory quality of cooked meatball samples

\begin{tabular}{|c|c|c|c|c|c|c|c|c|c|c|c|c|c|c|c|c|c|c|c|c|}
\hline \multicolumn{6}{|c|}{ Colour } & \multicolumn{5}{|c|}{ Smell } & \multicolumn{5}{|c|}{ Consistency } & \multicolumn{5}{|c|}{ Taste } \\
\hline 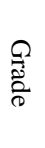 & 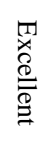 & 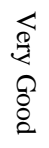 & $\begin{array}{l}8 \\
\stackrel{2}{2}\end{array}$ & 蒿 & 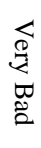 & $\frac{\delta}{\bar{g}}$ & 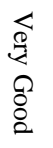 & $\begin{array}{l}\text { Q } \\
\varnothing \\
\vdots\end{array}$ & $\underset{\tilde{\tilde{D}}}{\mathscr{W}}$ & 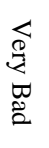 & 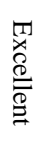 & 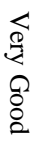 & $\begin{array}{l}0 \\
\stackrel{8}{\circ}\end{array}$ & 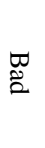 & 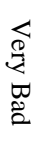 & 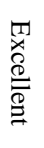 & 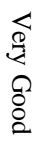 & $\begin{array}{l}8 \\
\stackrel{2}{2}\end{array}$ & $\begin{array}{l}\not{D} \\
\stackrel{0}{0}\end{array}$ & 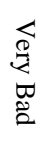 \\
\hline @ & $\vec{a}$ & $\stackrel{\infty}{\infty}$ & $\vec{a}$ & $\stackrel{8}{\circ}$ & $\stackrel{8}{\circ}$ & مै & $\begin{array}{l}\text { wa } \\
\text { ò }\end{array}$ & 高 & 㠃 & के & ¿ & वे & $\begin{array}{l}\text { aे } \\
\text { aे }\end{array}$ & के & \&े & $\vec{\circ}$ & 岁 & 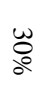 & $\begin{array}{l}\omega \\
o\end{array}$ & \&े \\
\hline in & $\begin{array}{l}8 \\
8 \\
3\end{array}$ & $\begin{array}{l}\text { Wa } \\
\text { ov }\end{array}$ & $\begin{array}{l}\text { wa } \\
\text { of }\end{array}$ & $\begin{array}{l}\text { 常 } \\
\partial^{2}\end{array}$ & 8 & $\frac{8}{9}$ & $\begin{array}{l}t \\
\text { a }\end{array}$ & के & 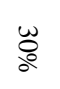 & 㠃 & $\stackrel{8}{\circ}$ & 亏े & $\begin{array}{l}\text { 㕝 } \\
\partial^{2}\end{array}$ & $\begin{array}{l}w \\
\text { a } \\
a\end{array}$ & 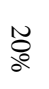 & $\begin{array}{l}8 \\
0 \\
0\end{array}$ & 产 & 产 & 裵 & $\begin{array}{l}\text { Oa } \\
\text { OQ }\end{array}$ \\
\hline$a^{9}$ & $\stackrel{8}{\circ}$ & के & 䐱 & वे & $\partial^{9}$ & $\partial^{9}$ & $\begin{array}{l}w \\
\partial Q\end{array}$ & प̆ & वे & 8 & $\stackrel{8}{\circ}$ & पू & $\begin{array}{l}\omega \\
\partial Q\end{array}$ & 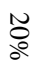 & $\begin{array}{l}8 \\
89\end{array}$ & $\stackrel{8}{\circ}$ & 产 & 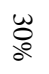 & $\begin{array}{l}\text { 常 } \\
\text { of }\end{array}$ & $\frac{8}{2}$ \\
\hline $\mathfrak{a}$ & $\stackrel{8}{\circ}$ & 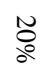 & 䐱 & 产 & a9 & $\begin{array}{l}8 \\
0 \\
0\end{array}$ & के & 崖 & oे & ¿े & $\stackrel{8}{\circ}$ & $\overbrace{0}^{\omega}$ & ò & 䐱 & ¿े & $\vec{\circ}$ & 产 & 产 & 网 & $\vec{a}$ \\
\hline $\begin{array}{l}w \\
a\end{array}$ & $\stackrel{8}{\circ}$ & के & 产 & $\begin{array}{l}1 \\
\text { aे } \\
\text { d }\end{array}$ & वे & a & $\begin{array}{l}\vec{t} \\
\text { da }\end{array}$ & $\begin{array}{l}\text { ta } \\
\text { a }\end{array}$ & $\frac{8}{8}$ & के & 高 & 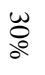 & $\begin{array}{l}1 \\
\text { aे } \\
\partial^{2}\end{array}$ & के & 高 & के & पू & 䐱 & $\begin{array}{l}\vec{b} \\
\text { a }\end{array}$ & $\vec{b}$ \\
\hline $\begin{array}{l}\text { a } \\
\text { a }\end{array}$ & $\stackrel{8}{a}$ & $\vec{a}$ & 㠃 & 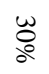 & & & 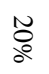 & 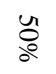 & $\begin{array}{l}\tilde{a} \\
\partial\end{array}$ & $\overbrace{}^{8}$ & $\frac{8}{9}$ & के & 岁 & o & के & 商 & 产 & $\begin{array}{l}\text { 常 } \\
\text { a }\end{array}$ & $\vec{\circ}$ & 8 \\
\hline
\end{tabular}


Table 3: Mean values of total aerobic colony count of meatball Samples incorporated with different concentration of Spirulina platensis

\begin{tabular}{|c|c|c|c|c|c|c|c|}
\hline & Control & $0.5 \%$ & $1.0 \%$ & $1.5 \%$ & $2.0 \%$ & $3.0 \%$ & $5.0 \%$ \\
\hline $\begin{array}{l}\text { days of } \\
\text { storage }\end{array}$ & $\begin{array}{c}\text { Mean } \\
\pm \\
\text { S.E. }\end{array}$ & $\begin{array}{c}\text { Mean } \\
\pm \\
\text { S.E. }\end{array}$ & $\begin{array}{c}\text { Mean } \\
\pm \\
\text { S.E. }\end{array}$ & $\begin{array}{c}\text { Mean } \\
\pm \\
\text { S.E. }\end{array}$ & $\begin{array}{c}\text { Mean } \\
\pm \\
\text { S.E. }\end{array}$ & $\begin{array}{c}\text { Mean } \\
\pm \\
\text { S.E. }\end{array}$ & $\begin{array}{c}\text { Mean } \\
\pm \\
\text { S.E. }\end{array}$ \\
\hline 0 & $\begin{array}{c}2.7 \times 10^{5} \\
\pm \\
1.4 \times 10^{5}\end{array}$ & $\begin{array}{c}2.2 \times 10^{5} \\
\pm \\
1.6 \times 10^{5}\end{array}$ & $\begin{array}{c}2.4 \times 10^{5} \\
\pm \\
1.7 \times 10^{5}\end{array}$ & $\begin{array}{c}2.6 \times 10^{5} \\
\pm \\
1.7 \times 10^{5}\end{array}$ & $\begin{array}{c}2.8 \times 10^{5} \\
\pm \\
2.3 \times 10^{5}\end{array}$ & $\begin{array}{c}2.8 \times 10^{5} \\
\pm \\
1.2 \times 10^{5}\end{array}$ & $\begin{array}{c}2.7 \times 10^{5} \\
\pm \\
1.2 \times 10^{5}\end{array}$ \\
\hline 2 & $\begin{array}{c}4.2 \times 10^{5} \\
\pm \\
2.2 \times 10^{5}\end{array}$ & $\begin{array}{c}2.6 \times 10^{5} \\
\pm \\
1.9 \times 10^{5}\end{array}$ & $\begin{array}{c}2.9 \times 10^{5} \\
\pm \\
2.0 \times 10^{5}\end{array}$ & $\begin{array}{c}3.7 \times 10^{5} \\
\pm \\
2.0 \times 10^{5}\end{array}$ & $\begin{array}{c}3 \times 10^{5} \\
\pm \\
2.4 \times 10^{5}\end{array}$ & $\begin{array}{c}3.2 \times 10^{5} \\
\pm \\
1.4 \times 10^{5}\end{array}$ & $\begin{array}{c}2.7 \times 10^{5} \\
\pm \\
1.2 \times 10^{5}\end{array}$ \\
\hline 3 & $\begin{array}{c}1 \times 10^{6} \\
\pm \\
5.2 \times 10^{5}\end{array}$ & $\begin{array}{c}5.8 \times 10^{5} \\
\pm \\
2.6 \times 10^{5}\end{array}$ & $\begin{array}{c}5.6 \times 10^{5} \\
\pm \\
2.6 \times 10^{5}\end{array}$ & $\begin{array}{c}4.6 \times 10^{5} \\
\pm \\
2.4 \times 10^{5}\end{array}$ & $\begin{array}{c}4.5 \times 10^{5} \\
\pm \\
2.6 \times 10^{5}\end{array}$ & $\begin{array}{c}3.7 \times 10^{5} \\
\pm \\
1.6 \times 10^{5}\end{array}$ & $\begin{array}{c}2.7 \times 10^{5} \\
\pm \\
1.2 \times 10^{5}\end{array}$ \\
\hline 4 & $\begin{array}{c}2.7 \times 10^{6} \\
\pm \\
1.3 \times 10^{6}\end{array}$ & $\begin{array}{c}2.4 \times 10^{6} \\
\pm \\
1.0 \times 10^{6}\end{array}$ & $\begin{array}{c}2.7 \times 10^{6} \\
\pm \\
1.2 \times 10^{6}\end{array}$ & $\begin{array}{c}2.3 \times 10^{6} \\
\pm \\
1.2 \times 10^{5}\end{array}$ & $\begin{array}{c}4.2 \times 10^{6} \\
\pm \\
2.5 \times 10^{5}\end{array}$ & $\begin{array}{c}6.5 \times 10^{5} \\
\pm \\
2.6 \times 10^{5}\end{array}$ & $\begin{array}{c}3.6 \times 10^{5} \\
\pm \\
1.6 \times 10^{5}\end{array}$ \\
\hline 6 & $\begin{array}{c}1.3 \times 10^{7} \\
\pm \\
6 \times 10^{6}\end{array}$ & $\begin{array}{c}1 \times 10^{7} \\
\pm \\
5.4 \times 10^{6}\end{array}$ & $\begin{array}{c}1.1 \times 10^{7} \\
\pm \\
5.9 \times 10^{6}\end{array}$ & $\begin{array}{c}1 \times 10^{7} \\
\pm \\
5.5 \times 10^{5}\end{array}$ & $\begin{array}{c}8.3 \times 10^{6} \\
\pm \\
5.0 \times 10^{5}\end{array}$ & $\begin{array}{c}4.7 \times 10^{6} \\
\pm \\
2.3 \times 10^{5}\end{array}$ & $\begin{array}{c}6.6 \times 10^{5} \\
\pm \\
2.9 \times 10^{5}\end{array}$ \\
\hline 8 & $\begin{array}{c}1.5 \times 10^{8} \\
\pm \\
7.9 \times 10^{7}\end{array}$ & $\begin{array}{c}6.9 \times 10^{7} \\
\pm \\
2.5 \times 10^{7}\end{array}$ & $\begin{array}{c}8.5 \times 10^{7} \\
\pm \\
3.9 \times 10^{7}\end{array}$ & $\begin{array}{c}7.1 \times 10^{7} \\
\pm \\
2.9 \times 10^{5}\end{array}$ & $\begin{array}{c}5.6 \times 10^{7} \\
\pm \\
2.6 \times 10^{5}\end{array}$ & $\begin{array}{c}6.1 \times 10^{6} \\
\pm \\
2.7 \times 10^{5}\end{array}$ & $\begin{array}{c}1.9 \times 10^{6} \\
\pm \\
1.1 \times 10^{5}\end{array}$ \\
\hline 9 & $\begin{array}{c}4.9 \times 10^{8} \\
\pm \\
2.4 \times 10^{8}\end{array}$ & $\begin{array}{c}1.8 \times 10^{8} \\
\pm \\
1.1 \times 10^{8}\end{array}$ & $\begin{array}{c}1.8 \times 10^{8} \\
\pm \\
8.7 \times 10^{7}\end{array}$ & $\begin{array}{c}1.3 \times 10^{8} \\
\pm \\
5.3 \times 10^{5}\end{array}$ & $\begin{array}{c}9.5 \times 10^{7} \\
\pm \\
4.0 \times 10^{5}\end{array}$ & $\begin{array}{c}1.7 \times 10^{7} \\
\pm \\
1.2 \times 10^{5}\end{array}$ & $\begin{array}{c}4 \times 10^{6} \\
\pm \\
2.2 \times 10^{5}\end{array}$ \\
\hline
\end{tabular}


Kareman S. Awadalla et al......

Table 4: Mean values of Enterobacteriaceae spp. count of meatball samples incorporated with different concentration of Spirulina platensis

\begin{tabular}{|c|c|c|c|c|c|c|c|}
\hline & Control & $0.5 \%$ & $1.0 \%$ & $1.5 \%$ & $2.0 \%$ & $3.0 \%$ & $5.0 \%$ \\
\hline days of & $\begin{array}{c}\text { Mean } \\
\pm\end{array}$ & $\begin{array}{c}\text { Mean } \\
\pm\end{array}$ & $\begin{array}{c}\text { Mean } \\
\pm\end{array}$ & $\begin{array}{c}\text { Mean } \\
\pm\end{array}$ & $\begin{array}{c}\text { Mean } \\
\pm\end{array}$ & $\begin{array}{c}\text { Mean } \\
\pm\end{array}$ & $\begin{array}{c}\text { Mean } \\
\pm\end{array}$ \\
\hline storage & S.E. & S.E. & S.E. & S.E. & S.E. & S.E. & S.E. \\
\hline 0 & $\begin{array}{c}2.2 \times 10^{4} \\
\pm \\
5.5 \times 10^{3}\end{array}$ & $\begin{array}{c}2.2 \times 10^{4} \\
\pm \\
6 \times 10^{3}\end{array}$ & $\begin{array}{c}3.8 \times 10^{4} \\
\pm \\
9.6 \times 10^{3}\end{array}$ & $\begin{array}{c}2.8 \times 10^{4} \\
\pm \\
5.6 \times 10^{3}\end{array}$ & $\begin{array}{c}3.9 \times 10^{4} \\
\pm \\
6.6 \times 10^{2}\end{array}$ & $\begin{array}{c}2.9 \times 10^{4} \\
\pm \\
4.9 \times 10^{3}\end{array}$ & $\begin{array}{c}7.7 \times 10^{3} \\
\pm \\
1.2 \times 10^{3}\end{array}$ \\
\hline 2 & $\begin{array}{c}3.1 \times 10^{4} \\
\pm \\
4.3 \times 10^{3}\end{array}$ & $\begin{array}{c}3.2 \times 10^{4} \\
\pm \\
4.4 \times 10^{3}\end{array}$ & $\begin{array}{c}4.1 \times 10^{4} \\
\pm \\
8.7 \times 10^{3}\end{array}$ & $\begin{array}{c}3.5 \times 10^{4} \\
\pm \\
2.9 \times 10^{3}\end{array}$ & $\begin{array}{c}4.2 \times 10^{4} \\
\pm \\
4.6 \times 10^{3}\end{array}$ & $\begin{array}{c}1.8 \times 10^{4} \\
\pm \\
4.4 \times 10^{3}\end{array}$ & $\begin{array}{c}2.4 \times 10^{3} \\
\pm \\
8.1 \times 10^{2}\end{array}$ \\
\hline 3 & $\begin{array}{c}8.6 \times 10^{4} \\
\pm \\
6.7 \times 10^{3}\end{array}$ & $\begin{array}{c}2.5 \times 10^{4} \\
\pm \\
2.9 \times 10^{3}\end{array}$ & $\begin{array}{c}2.7 \times 10^{4} \\
\pm \\
6 \times 10^{3}\end{array}$ & $\begin{array}{c}2.5 \times 10^{4} \\
\pm \\
2.9 \times 10^{3}\end{array}$ & $\begin{array}{c}2.3 \times 10^{4} \\
\pm \\
3.3 \times 10^{3}\end{array}$ & $\begin{array}{c}4 \times 10^{3} \\
\pm \\
1.7 \times 10^{3}\end{array}$ & $\begin{array}{c}1.7 \times 10^{3} \\
\pm \\
4.4 \times 10^{2}\end{array}$ \\
\hline 4 & $\begin{array}{c}1 \times 10^{5} \\
\pm \\
9.3 \times 10^{3}\end{array}$ & $\begin{array}{c}2 \times 10^{4} \\
\pm \\
2.9 \times 10^{3}\end{array}$ & $\begin{array}{c}1.5 \times 10^{4} \\
\pm \\
2.9 \times 10^{3}\end{array}$ & $\begin{array}{c}1.2 \times 10^{4} \\
\pm \\
1.4 \times 10^{3}\end{array}$ & $\begin{array}{c}1.3 \times 10^{4} \\
\pm \\
2.2 \times 10^{3}\end{array}$ & $\begin{array}{c}1.6 \times 10^{3} \\
\pm \\
7 \times 10^{2}\end{array}$ & $\begin{array}{c}7 \times 10^{1} \\
\pm \\
1.5 \times 10^{1}\end{array}$ \\
\hline 6 & $\begin{array}{c}2.6 \times 10 \\
\pm \\
1.4 \times 10^{4}\end{array}$ & $\begin{array}{c}1.4 \times 10^{4} \\
\pm \\
2 \times 10^{3}\end{array}$ & $\begin{array}{c}1.1 \times 10^{3} \\
\pm \\
1.8 \times 10^{3}\end{array}$ & $\begin{array}{c}6.3 \times 10^{3} \\
\pm \\
1.4 \times 10^{3}\end{array}$ & $\begin{array}{c}5.3 \times 10^{3} \\
\pm \\
1.8 \times 10^{3}\end{array}$ & $\begin{array}{c}1.4 \times 10^{3} \\
\pm \\
5.4 \times 10^{2}\end{array}$ & $\begin{array}{c}2.6 \times 10^{1} \\
\pm \\
6.6 \times 10^{0}\end{array}$ \\
\hline 8 & $\begin{array}{c}3.8 \times 10^{5} \\
\pm \\
6 \times 10^{4}\end{array}$ & $\begin{array}{c}1.4 \times 10^{5} \\
\pm \\
2.1 \times 10^{4}\end{array}$ & $\begin{array}{c}7.3 \times 10^{3} \\
\pm \\
1.4 \times 10^{3}\end{array}$ & $\begin{array}{c}3.7 \times 10^{3} \\
\pm \\
1.4 \times 10^{3}\end{array}$ & $\begin{array}{c}2.7 \times 10^{3} \\
\pm \\
1.2 \times 10^{3}\end{array}$ & $\begin{array}{c}1 \times 10^{3} \\
\pm \\
2.3 \times 10^{2}\end{array}$ & $\begin{array}{c}1.3 \times 10^{1} \\
\pm \\
3.3 \times 10^{0}\end{array}$ \\
\hline 9 & $\begin{array}{c}6.7 \times 10^{5} \\
\pm \\
3.3 \times 10^{4}\end{array}$ & $\begin{array}{c}3.6 \times 10^{5} \\
\pm \\
6.9 \times 10^{4}\end{array}$ & $\begin{array}{c}3 \times 10^{3} \\
\pm \\
1.1 \times 10^{3}\end{array}$ & $\begin{array}{c}1.3 \times 10^{3} \\
\pm \\
3.7 \times 10^{2}\end{array}$ & $\begin{array}{c}9.7 \times 10^{2} \\
\pm \\
4.6 \times 10^{2}\end{array}$ & $\begin{array}{c}8.3 \times 10^{2} \\
\pm \\
8.8 \times 10^{1}\end{array}$ & $\begin{array}{c}1 \times 10^{1} \\
\pm \\
1 \times 10^{0}\end{array}$ \\
\hline
\end{tabular}


Table 5: Mean values of Staphylococcus aureus count of meatball samples incorporated with different concentration of Spirulina platensis

\begin{tabular}{|c|c|c|c|c|c|c|c|}
\hline \multirow{3}{*}{$\begin{array}{l}\text { Days of } \\
\text { storage }\end{array}$} & Control & $0.5 \%$ & $1.0 \%$ & $1.5 \%$ & $2.0 \%$ & $3.0 \%$ & $5.0 \%$ \\
\hline & $\begin{array}{c}\text { Mean } \\
\pm\end{array}$ & $\begin{array}{c}\text { Mean } \\
\pm\end{array}$ & $\begin{array}{c}\text { Mean } \\
\pm\end{array}$ & $\begin{array}{c}\text { Mean } \\
\pm\end{array}$ & $\begin{array}{c}\text { Mean } \\
\pm\end{array}$ & $\begin{array}{c}\text { Mean } \\
\pm\end{array}$ & $\begin{array}{c}\text { Mean } \\
\pm\end{array}$ \\
\hline & S.E. & S.E. & S.E. & S.E. & S.E. & S.E. & S.E. \\
\hline \multirow{3}{*}{0} & $2.3 \times 10^{4}$ & $2 \times 10^{4}$ & $1.6 \times 10^{4}$ & $1.7 \times 10^{4}$ & $1.4 \times 10^{4}$ & $1.6 \times 10^{4}$ & $1.1 \times 10^{4}$ \\
\hline & \pm & \pm & \pm & \pm & \pm & \pm & \pm \\
\hline & $3.7 \times 10^{3}$ & $2.6 \times 10^{3}$ & $4.2 \times 10^{3}$ & $3.8 \times 10^{3}$ & $3.2 \times 10^{3}$ & $3.4 \times 10^{3}$ & $3.2 \times 10^{3}$ \\
\hline \multirow{3}{*}{2} & $6.2 \times 10^{4}$ & $5 \times 10^{4}$ & $3.9 \times 10^{4}$ & $3.5 \times 10^{4}$ & $3.2 \times 10^{4}$ & $2.7 \times 10^{4}$ & $1.9 \times 10^{4}$ \\
\hline & \pm & 1 & $\stackrel{-}{4}$ & \pm & \pm & \pm & \pm \\
\hline & $1.9 \times 10^{4}$ & $1.1 \times 10^{7}$ & $1 \times 10^{\circ}$ & $7.6 \times 10^{5}$ & $7.5 \times 10^{\circ}$ & $4.6 \times 10^{3}$ & $2.3 \times 10^{3}$ \\
\hline \multirow{3}{*}{3} & $2.8 \times 10^{5}$ & $1.9 \times 10^{5}$ & $1.5 \times 10^{5}$ & $9.5 \times 10^{4}$ & $6.3 \times 10^{4}$ & $4.8 \times 10^{4}$ & $3.4 \times 10^{4}$ \\
\hline & + & \pm & \pm & \pm & \pm & \pm & \pm \\
\hline & $1.2 \times 10^{5}$ & $7 \times 10^{4}$ & $5.9 \times 10$ & $3.3 \times 10^{4}$ & $2.2 \times 10^{4}$ & $1.7 \times 10^{4}$ & $8.9 \times 10^{3}$ \\
\hline \multirow{3}{*}{4} & $5.8 \times 10^{5}$ & $2.6 \times 10^{5}$ & $3.8 \times 10^{5}$ & $2.7 \times 10^{5}$ & $1.4 \times 10^{5}$ & $9.9 \times 10^{4}$ & $7.9 \times 10^{4}$ \\
\hline & \pm & \pm & \pm & \pm & \pm & \pm & \pm \\
\hline & $2.4 \times 10^{5}$ & $8.8 \times 10^{4}$ & $1.5 \times 10^{5}$ & $9.8 \times 10^{4}$ & $3.8 \times 10^{4}$ & $2.2 \times 10^{4}$ & $1.5 \times 10^{4}$ \\
\hline \multirow{3}{*}{6} & $1 \times 10^{6}$ & $6.2 \times 10^{5}$ & $5.7 \times 10^{5}$ & $5.2 \times 10^{5}$ & $2 \times 10^{5}$ & $1.7 \times 10^{5}$ & $1.2 \times 10^{5}$ \\
\hline & \pm & \pm & \pm & \pm & \pm & \pm & \pm \\
\hline & $2.9 \times 10^{5}$ & $1.3 \times 10^{5}$ & $1.6 \times 10^{5}$ & $1.6 \times 10^{5}$ & $2.9 \times 10^{4}$ & $3.5 \times 10^{4}$ & $2.3 \times 10^{4}$ \\
\hline \multirow{3}{*}{8} & $2.2 \times 10^{6}$ & $1.6 \times 10^{6}$ & $1.1 \times 10^{6}$ & $1.1 \times 10^{6}$ & $6.5 \times 10^{5}$ & $2.8 \times 10^{5}$ & $1.6 \times 10^{5}$ \\
\hline & + & \pm & \pm & \pm & \pm & \pm & \pm \\
\hline & $\begin{array}{c} \pm \\
4.4 \times 10^{5}\end{array}$ & $2.3 \times 10^{5}$ & $1.8 \times 10^{5}$ & $1.8 \times 10^{5}$ & $1.2 \times 10^{5}$ & $6 \times 10^{4}$ & $2.9 \times 10^{4}$ \\
\hline \multirow{3}{*}{9} & $6.9 \times 10^{6}$ & $2.9 \times 10^{6}$ & $1.8 \times 10^{6}$ & $1.7 \times 10^{6}$ & $9.5 \times 10^{5}$ & $3.4 \times 10^{5}$ & $1.5 \times 10^{5}$ \\
\hline & \pm & \pm & \pm & \pm & \pm & \pm & \pm \\
\hline & $1.5 \times 10^{6}$ & $3.5 \times 10^{5}$ & $3.6 \times 10^{5}$ & $3.9 \times 10^{5}$ & $2.9 \times 10^{4}$ & $8 \times 10^{4}$ & $2.9 \times 10^{4}$ \\
\hline
\end{tabular}


Kareman S. Awadalla et al......

Figure 1: Effect of addition of Spirulina platensis with different concentrations on color of raw meatball samples

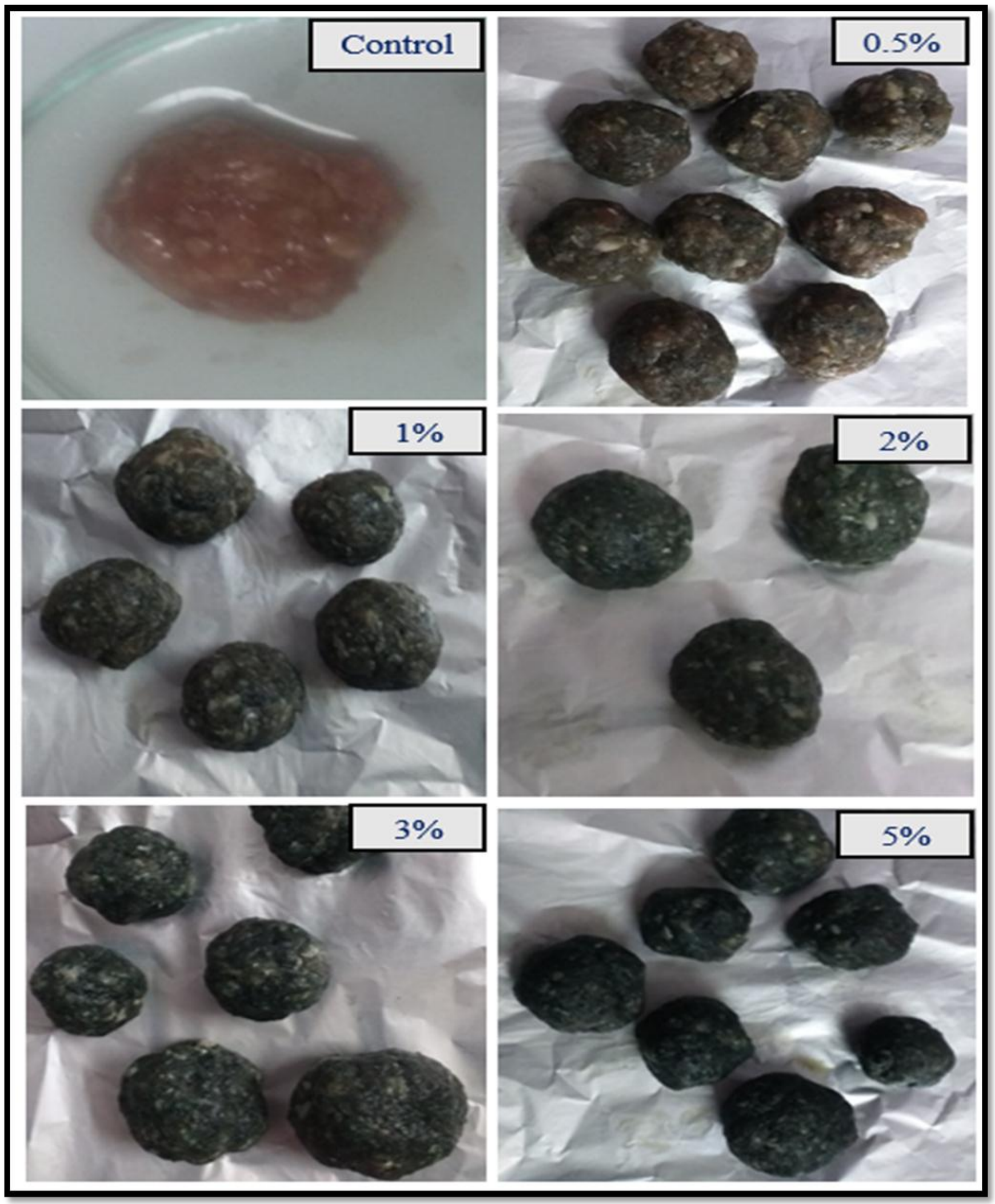




\section{DISCUSSION}

The use of antimicrobial ingredients is one of the widely used methods to maintain microbiological safety and prolong the shelf life of food products (Aiada, et al., 2017). Shelf-life of raw meat is limited by microbial spoilage activity. Meat products with natural antimicrobial preservatives are demanded by consumers. The smart application of hurdle technology became more dominant, because the principles of major preservative factors for foods (e.g., temperature, $\mathrm{pH}, a \mathrm{w}$, preservatives), and their interactions, became better known (Leistner, 2000). Combinations of low temperature with other antimicrobial ingredients may halt the growth of such pathogens so, predictive microbiology and specific growth/no growth interface models are effective tools for identifying such combinations (McMeekin et al., 2000).

\section{Sensory Evaluation}

As the sensorial characteristics of foods are of significant importance, their modification resulting from the addition or subtraction of components should be carefully studied in order to check the consumer reaction due to possible changes in product taste, texture, colour or odour. The taste, odour, colour and texture of the meatball raw and cooked samples incorporated with Spirulina platensis in different concentrations were evaluated. Colour is one of the most important quality properties for the acceptability of food due to its relation with product freshness and flavour expectations and therefore has a direct effect on consumers' perceptions.

Results are shown in Figure (1) declared the effect of addition of Spirulina platensis by different concentrations on colour of raw meatball samples as following; the control meatball sample had bright red acceptable colour, while addition of $0.5 \%$ Spirulina platensis to meatball gave it acceptable mild green colour which became more intense in samples mixed with $1 \%, 2 \%, 3 \%$, and $5 \%$ of Spirulina platensis causing a different acceptability range. The effect of the addition of Spirulina platensis with different concentrations to raw meatball samples on sensory quality was illustrated in Table (1) as follows: the colour of the examined control samples was 30\% excellent, $30 \%$ very good and $40 \%$ good. The addition of $0.5 \%$ Spirulina platensis showed that about $10 \%$ of the samples were very good, $10 \%$ good, $70 \%$ bad and $10 \%$ very bad.

It was declared that the color of the examined samples was $50 \%, 50 \%, 60 \%$ and $70 \%$ very bad and $30 \%, 30 \%, 30 \%$ and $20 \%$ bad by addition of $1 \%, 2 \%$, $3 \%$ and $5 \%$ Spirulina platensis respectively. Smell character of the examined control samples was $60 \%$ very good, while the highest value after addition of $0.5 \%$ Spirulina platens was; $40 \%$ good, $30 \%$ bad, $10 \%$ excellent, $10 \%$ very good and $10 \%$ very bad grade. On the other hand, $30 \%$ of the samples were very good when Spirulina platensis was added with concentrations of $1 \%, 2 \%, 3 \%$ and $5 \%$. The reported consistency of the meatball samples was $50 \%$ very good, $60 \%$ good, $50 \%$ very good, $50 \%$ good, $60 \%$ good and $40 \%$ good for control, $0.5 \%, 1 \%, 2 \%, 3 \%$ and $5 \%$ concentrations of Spirulina platensis respectively.

The effect of the addition of Spirulina platensis with different concentrations on sensory quality of cooked meatball samples was shown in Table (2). The color of cooked control meatball samples was $80 \%$ very good, while most of the samples were $40 \%, 60 \%$, $30 \% 40 \%$ good for $0.5 \%, 1 \%, 2 \%, 3 \%$ Spirulina platensis concentrations, respectively. The addition of $5 \%$ of Spirulina platensis resulted in $40 \%$ of cooked meatball samples had a very bad colour. Concerning the smell of cooked meatball samples, it was clear that control, $0.5 \%, 2 \%$ and 3\% concentrations of Spirulina platensis had a very good smell per cent $(30 \%),(40 \%)$, $(60 \%)$ and (40\%) respectively. While $50 \%, 30 \%, 40 \%$, $50 \%$ of cooked meat samples had a good smell for concentrations of $1 \%, 2 \%, 3 \%$ \& $5 \%$ Spirulina platensis respectively.

Furthermore, $50 \%, 50 \%, 30 \%, 30 \%$ of the cooked meat samples had a very good consistency for control, $1 \%, 2 \%$ and 3\% Spirulina platensis concentrations respectively, while $40 \%, 40 \%, 30 \%$, $50 \%, 40 \%$ and $50 \%$ of the samples were scored as good for consistency in control, $0.5 \%, 1 \%, 2 \%, 3 \%$ and $5 \%$ Spirulina platensis concentrations respectively. Additionally, $30 \%$ of the cooked meat samples were reported very good and good for taste in control, $0.5 \%$, $1 \%, 2 \%$ Spirulina platensis concentrations, whereas $40 \%$ of the samples were reported as good in case of addition of 5\% Spirulina platensis.

Results revealed that the effect of added Spirulina platensis concentrations on the colour of raw meatball samples was mainly unacceptable in comparison to control samples. However, the addition of different concentrations of Spirulina platensis to cooked meatball samples was more acceptable than raw samples. The changes in colour and flavour in foods are usually perceived as undesirable by consumers (Jeon, 2006, Prakash and Kumari, 2011 and Beheshtipour, et al., 2013). Becker (2007) and Fradique, et al. (2013) explained that the green colour of microalgae limits its application in daily-use products, as it adversely affects consumers' perception about taste and quality. Consumer rejection to this novel microalgae as food additives refereeing to the 
traditional social and moral factors while, acceptance of children is easier due to their trend for unusual preparing of foods with attractive colour and taste (Sajilata, et al., 2007).

The addition of Spirulina platensis had an adverse effect on the colour of raw meatball samples, but it gave acceptable smell and consistency for raw meatball samples. On the other hand, the colour of cooked meatball samples was adversely affected by the addition of Spirulina platensis, while the smell remained acceptable when the different concentration of Spirulina platensis was added. Consistency was mildly influenced by the addition of Spirulina platensis, especially at 5\% concentration. Furthermore, the taste was not affected by the addition of different concentrations of Spirulina platensis.

Similar results were reported by Enver, et al. (2012) who evaluated the sensorial acceptance of fresh pasta incorporated with 5\% Spirulina platensis and found that the sensorial quality was considered satisfactory. Özyurt et al. (2015) estimated the sensorial quality of pasta with the addition of Spirulina platensis at $5 \%$ and $10 \%$ concentrations and observed that the addition of Spirulina platensis is one of the best choices for coloured pasta because of the positive impact on the consumers. Sensory evaluation indicated that pasta enriched with S. platensis had a good overall score the same as the control group in terms of taste and consistency. Spirulina added nutritional and organoleptic (colour, odour \& taste) properties to noodles with strong elasticity.

\section{Bacteriological evaluation \\ 2.1 Aerobic Colony Count (ACC)}

Table (3) elucidated the total aerobic colony count of meatball samples at $(0,2,3,4,6,8$, and 9) days of refrigeration storage where the mean values of total colony count in control samples were $2.7 \times 10^{5}$, $4.2 \times 10^{5}, 1 \times 10^{6}, 2.7 \times 10^{6}, 1.3 \times 10^{7}, 1.5 \times 10^{8}$ and $4.9 \times 10^{8}$ CFU/g respectively. The obtained results revealed that microbial growth increased during refrigeration storage. The mean values of total colony count of examined meatball samples mixed with $0.5 \%$ Spirulina platensis were $2.2 \times 10^{5}, 2.6 \times 10^{5}, 5.8 \times 10,2.4 \times 10^{6}$, $1 \times 10^{7}, 6.9 \times 10^{7}$ and $1.8 \times 10^{8} \mathrm{CFU} / \mathrm{g}$ at $(0,2,3,4,6,8$, and 9) days of refrigeration storage respectively. The addition of $1 \%$ Spirulina platensis to meatball samples succeeded in reducing the total aerobic count where the estimated mean values were $2.4 \times 10^{5}, 2.9 \times 10^{5}, 5.6 \times 10^{5}$, $2.7 \times 10^{6}, 1.1 \times 10^{7}, 8.5 \times 10^{7}$ and $1.8 \times 10^{8} \mathrm{CFU} / \mathrm{g}$ at $(0,2$, $3,4,6,8$, and 9) days of refrigeration storage respectively.

The obtained mean values for total colony count after addition of $1.5 \%$ Spirulina platensis to meatball samples were $2.6 \times 10^{5}, 3.7 \times 10^{5}, 4.6 \times 10^{5}$, $2.3 \times 10^{6}, 1 \times 10^{7}, 7.1 \times 10^{7}$ and $1.3 \times 10^{8} \mathrm{CFU} / \mathrm{g}$ at $(0,2,3$, $4,6,8$, and 9) days of refrigeration storage respectively. The addition of $2 \%$ Spirulina platensis to meatball samples decreased to some extent the mean total aerobic count into $2.8 \times 10^{5}, 3 \times 10^{5}, 4.5 \times 10^{5}$, $4.2 \times 10^{6}, 8.3 \times 10^{6}, 5.6 \times 10^{7}$ and $9.5 \times 10^{7} \mathrm{CFU} / \mathrm{g}$ at $(0,2$, $3,4,6,8$, and 9) days of refrigeration storage respectively. The mean values of total aerobic count of examined meatball samples treated with $3 \%$ Spirulina platensis were $2.8 \times 10^{5}, 3.2 \times 10^{5}, 3.7 \times 10^{5}, 6.5 \times 10^{5}$, $4.7 \times 10^{6}, 6.1 \times 10^{6}$ and $1.7 \times 10^{7} \mathrm{CFU} / \mathrm{g}$ at $(0,2,3,4,6,8$, and 9) days of refrigeration storage respectively. The addition of 5\% Spirulina platensis to meatball samples resulted in a clear reduction of the mean total aerobic count into $2.7 \times 10^{5}, 2.7 \times 10^{5}, 2.7 \times 10^{5}, 3.6 \times 10^{5}, 6.6 \times 10^{5}$, $1.9 \times 10^{6}$ and $4 \times 10^{6} \mathrm{CFU} / \mathrm{g}$ at $(0,2,3,4,6,8$, and 9) days of refrigeration storage respectively. The obtained values were higher than the values recommended by EOS (1694/2005), which is $1 \times 10^{6} \mathrm{CFU} / \mathrm{g}$.

Spirulina has the ability to inhibit the growth of certain microorganisms; both pathogens that pose a health hazard, as well as those that cause food spoilage in all branches of the food industry (Kaushik and Chuhan, 2008). They reported that Spirulina platensis has an antimicrobial activity to reach an ultimate mean value of $4.9 \times 10^{8} \pm 2$. CFU /g after 9 days of storage in the refrigerator. Veli and Yasemin (2012) investigated the shelf life of meatball during storage and found that microbial count increased with time during the storage period $(P<0.0001)$, whereas, at the beginning of the storage, the mean value of the aerobic bacterial count of control samples was $2.4 \times 10^{4} \mathrm{CFU} / \mathrm{g}$, it increased after 10 days and reached $1.2 \times 10^{8} \mathrm{CFU} / \mathrm{g}$. According to Ailton et al. (2012), the examination of microbial count in traditional meatball increased during 24 hours of refrigeration storage; at zero hours microbial count was $7 \times 10^{5}$ which increased with time to $7.6 \times 10^{5}$ at 24 hours. Spirulina may be an alternative to the widely used chemical preservatives used in food technology.

\subsection{Enterobacteriaceae spp.}

Table (4) clarified the mean values of Enterobacteriaceae spp.count of meatball samples at $(0,2,3,4,6,8$, and 9$)$ days of refrigeration storage where the mean values of Enterobacteriaceae spp.count in control samples were $2.2 \times 10^{4}, 3.1 \times 10^{4}$, $8.6 \times 10^{4}, 1 \times 10^{5}, 2.6 \times 10^{5}, 3.8 \times 10^{5}$ and $6.7 \times 10^{5} \mathrm{CFU} / \mathrm{g}$ respectively. The mean values of Enterobacteriaceae spp.count of examined meatball samples mixed with $0.5 \%$ Spirulina platensis were $2.2 \times 10^{4}, 3.2 \times 10^{4}$, $2.5 \times 10^{4}, 2 \times 10^{4}, 1.4 \times 10^{4}, 1.4 \times 10^{5}$ and $3.6 \times 10^{5} \mathrm{CFU} / \mathrm{g}$ at $(0,2,3,4,6,8$, and 9$)$ days of refrigeration storage respectively. To a lesser extent the addition of $1 \%$ Spirulina platensis to meatball samples led to a redution in Enterobacteriaceae spp.count at days 6,8 
and 9 of storage where the estimated mean values were $3.8 \times 10^{4}, 4.1 \times 10^{4}, 2.7 \times 10^{4}, 1.5 \times 10^{4}, 1.1 \times 10^{4}, 7.3 \times 10^{3}$ and $3 \times 10^{3} \mathrm{CFU} / \mathrm{g}$ at $(0,2,3,4,6,8$, and 9) days of refrigeration storage respectively. The obtained mean values for Enterobacteriaceae spp.count after addition of $1.5 \%$ Spirulina platensis to meatball samples were $2.8 \times 10^{4}, 3.5 \times 10^{4}, 2.5 \times 10^{4}, 1.2 \times 10^{4}, 6.3 \times 10^{3}, 3.7 \times 10^{3}$ and $1.3 \times 10^{3} \mathrm{CFU} / \mathrm{g}$ after $(0,2,3,4,6,8$, and 9$)$ days of refrigeration storage respectively.

The addition of $2 \%$ Spirulina platensis to meatball samples decreased to some extent the mean of Enterobacteriaceae spp.count into $3.9 \times 10^{4}, 4.2 \times 10^{4}$, $2.3 \times 10^{4}, 1.3 \times 10^{4}, 5.3 \times 10^{3}, 2.7 \times 10^{3}$ and $9.7 \times 10^{2} \mathrm{CFU} / \mathrm{g}$ at $(0,2,3,4,6,8$, and 9$)$ days of refrigeration storage respectively in comparison with the lowest concentrations. The mean values of Enterobacteriaceae spp.count of examined meatball samples treated with $3 \%$ Spirulina platensis were obviously reduced to $2.9 \times 10^{4}, 1.8 \times 10^{4}, 4 \times 10^{3}, 1.6 \times 10^{3}, 1.4 \times 10^{3}, 1 \times 10^{3}$ and $8.3 \times 10^{2} \mathrm{CFU} / \mathrm{g}$ at $(0,2,3,4,6,8$, and 9) days of refrigeration storage respectively. The addition of $5 \%$ Spirulina platensis to meatball samples resulted in a clear reduction of the Enterobacteriaceae spp.count into $7.7 \times 10^{3}, 2.4 \times 10^{3}, 1.7 \times 10^{3}, 7 \times 10^{1}, 2.6 \times 10^{1}, 1.3 \times 10^{1}$ and $1 \times 10^{1} \mathrm{CFU} / \mathrm{g}$ at $(0,2,3,4,6,8$, and 9) days of refrigeration storage of samples respectively.

The mean values of Enterobacteriaceae spp.count were significantly increased $(p>0.05)$ with extending the storage time.Veli and Yasemin, (2012) investigated the shelf life of meatball during storage and found that microbial count increased with time during the storage period $(P<0.0001)$, whereas at the beginning of the storage, the mean value of Enterobacteriaceae spp. count of control samples was $6.3 \times 10^{3} \mathrm{CFU} / \mathrm{g}$, which increased after 10 days to $1.9 \mathrm{x}$ $10^{7} \mathrm{CFU} / g$. Duda-Chodak (2013) found that Spirulina platensis water extract had no impact on E.coli. On the other hand, the methanolic extract of Spirulina platensis had antimicrobial activity against some spp. of Enterobacteriaceae; as E.coli, Salmonella typhi, Klebsiella pneumoniae and Shigella flexneri (Kaushik and Chauhan, 2008 and Usharani et al., 2015).

\subsection{Staphylococcus aureus Count}

The mean values of Staphylococcus aureus count of meatball samples incorporated with different concentration of Spirulina platensis at $(0,2,3,4,6,8$, and 9) days of refrigeration storage were shown in Table (5) where the obtained mean values of the Staphylococcus aureus count in control samples were $2.3 \times 10^{4}, 6.2 \times 10^{4}, 2.8 \times 10^{5}, 5.8 \times 10^{6}, 1 \times 10^{6}, 2.2 \times 10^{6}$ and $6.9 \times 10^{6} \mathrm{CFU} / \mathrm{g}$ respectively. There was a clear reduction in Staphylococcus aureus count in meatball samples treated with $0.5 \%\left(2 \times 10^{4}, 5 \times 10^{4}, 1.9 \times 10^{5}\right.$, $2.6 \times 10^{5}, 6.2 \times 10^{5}, 1.6 \times 10^{6}$ and $\left.2.9 \times 10^{6} \mathrm{CFU} / \mathrm{g}\right), 1 \%$ $\left(1.6 \times 10^{4}, 3.9 \times 10^{4}, 1.5 \times 10^{5}, 3.8 \times 10^{5}, 5.7 \times 10^{5}, 1.1 \times 10^{6}\right.$, $\left.1.8 \times 10^{6} \mathrm{CFU} / \mathrm{g}\right), 1.5 \%\left(1.7 \times 10^{4}, 3.5 \times 10^{4}, 9.5 \times 10^{4}\right.$, $2.7 \times 10^{5} \pm, 5.2 \times 10^{5}, 1.1 \times 10^{6}$ and $\left.1.7 \times 10^{6} \mathrm{CFU} / \mathrm{g}\right), 2 \%$ $\left(1.4 \times 10^{4}, 3.2 \times 10^{4}, 6.3 \times 10^{4}, 1.4 \times 10^{5}, 2 \times 10^{5}, 6.5 \times 10^{5}\right.$ and $\left.9.5 \times 10^{5} \quad \mathrm{CFU} \quad / \mathrm{g}\right), \quad 3 \% \quad\left(1.6 \times 10^{4}, \quad 2.7 \times 10^{4}\right.$, $4.8 \times 10^{4}, 9.9 \times 10^{4}, 1.7 \times 10^{5}, 2.8 \times 10^{5}$ and $\left.3.4 \times 10^{5} \mathrm{CFU} / \mathrm{g}\right)$ and $5 \%$ Spirulina platensis $\left(1.1 \times 10^{4}, 1.9 \times 10^{4}\right.$, $3.4 \times 10^{4}, 7.9 \times 10^{4}, 1.2 \times 10^{5}, 1.6 \times 10^{5}$ and $1.5 \times 10^{5} \mathrm{CFU} / \mathrm{g}$ ) at $(0,2,3,4,6,8$, and 9) days of refrigeration storage respectively. The mean values of Staphylococcus aureus count were significantly increased $(p>0.05)$ with extending the storage time. The extract of Spirulina platensis had antimicrobial activity against Staph. Aureus (Kaushik and Chauhan, 2008, Kumar et al., 2011 and Usharani et al., 2015).

\section{CONCLUSION}

The addition of Spirulina platensis had an adverse effect on the colour of raw meatball samples but it gave acceptable smell and consistency. On the other hand, the colour of cooked meatball samples was adversely affected by the addition of Spirulina platensis, while smell remained acceptable when different concentrations of Spirulina platensis were used. Consistency was mildly affected by the addition of Spirulina platensis, especially at 5\% concentration. Furthermore, the taste was not affected by different concentrations of Spirulina platensis. It was clear that microbial growth increased during refrigeration storage, while the addition of Spirulina platensis reduced total aerobic colony, Enterobacteriaceae spp. and Staphylococcus aureus counts. The study recommended enhancements of further studies concerning the use of Spirulina platensis in different meat product as natural preservatives.

\section{REFERENCES}

ABD El-MALEK A. M. 2017. Bacteriological status and detection of adulteration with donkey meat by PCR in frozen beef meatballs, IOSR Journal of Environmental Science, Toxicology and Food Technology, Vol.11 (10): 1-7.

AIADA D. M., RAMADAN D. M., HAOW M. M. AND SUMAYYAH A. A. 2017. Microbiological quality of fresh and frozen ground meat, Alexandria city, Egypt. Saudi Journal of Pathology and Microbiology, Vol-2, Iss-9:286-290.

AILTON C. L., KATIUCHIA P. T., JOÃO C. M. C., ELIANE D. AND GODOY D. 2012. Fresh pasta production enriched with Spirulina platensis biomass. Braz. arch. biol. technol. vol.55 (.5)..741-750.

BALCH P A. AND BALCH J.F. 2000. Prescription for nutritional healing. Part I, Natural Food Supplements, Health and Fitness. $3^{\text {rd }}$ Edition pp. 776.

BECKER E.W.2007. Micro-algae as a source of protein. Biotechnol. Adv. 25, 207-210. 
BEHESHTIPOUR H., MORTAZAVIAN A.M., MOHAMMADI R., SOHRABVANDI S. AND KHOSRAVI-DARANI K. 2013. Supplementation of Spirulina platensis and Chlorella Vulgaris algae into probiotic fermented milk. Comp. Rev. Food Sci. Food Safety. 12 (2), 144-154.

DIMPLE M.C.Z., DECAIRE G.Z. AND DECANO M.S. 1996. Bioactive substances from Spirulina platensis (Cyanobacteria). International Journal of Experimental Botany; 58: 93-96.

DUDA-CHODAK A. 2013. Impact of water extracts of Spirulina (WES) on bacteria, yeasts and moulds. Acta Sci. Pol., Technol. Aliment. 12(1):33-39

EGYPTIAN ORGANIZATION FOR STANDARDIZATION AND QUALITY CONTROL. 2005. Egyptian standards froze minced meat, Egypt, E.S 1694.

ENVER B. B., HITAL C., OMER C. AND HAMPARSUN H. 2012. Effects of sodium lactate on the shelf life and sensory characteristics of CIG KOFTE - A Turkish traditional raw meatball. Journal of Food Processing and Preservation, (38): 10241036.

FAO. 2015. Meat and meat products in human nutrition in developing countries. Available at http://www.fao.org/ag/againfo/themes/en/meat/backgr ound.html. Accessed on 12/02/2015

FRADIQUE M., BATISTA A.P., NUNES M.C., GOUVEIA L., BANDARRA N.M.AND RAYMUNDO A. 2013. Isochrysis galbana and Diacronema vlkianum biomass incorporation in pasta products as PUFA's source. LWT J Food Sci Technol, 50:312-319.

GHAENI M. AND ROOMIANI L. 2016. Review for Application and Medicine Effects of Spirulina, Microalgae. Journal of Advanced Agricultural Technologies. 3(2): 1-3.

GRAPH PAD INSTANT. 2009. GraphPad Instant Software, Inc.

HOSSEINI S.M., SHAHBAZIZADEH, S., KHOSRAVIDARANI, K. AND MOZAFARI, M.R. 2103. Spirulina paltensis: Food and Function. Current Nutrition \& Food Science. 9(2): 1-5.

INTERNATIONAL COMMISSION OF MICROBIOLOGICAL SPECIFICATION FOR FOODS "ICMSF" .1996. Microorganisms in Food. I-Their Significance and methods of enumeration.3rd Ed. Univ. of Toronto, Canada.

INTERNATIONAL ORGANIZATION FOR STANDARDIZATION "ISO". 2004. No. 11291-1. Microbiology of food and animal feeding stuffs Horizontal methods for detection and enumeration of Enterobacteriaceae part 2: colony count method.

JEON J.K. 2006. Effect of Chlorella addition on the quality of processed cheese. J. Korean Soc. Food Sci. Nutr., 35 (3):373-377.

KAUSHIK P. AND CHAUHAN A. 2008. In vitro antibacterial activity of a laboratory-grown culture of Spirulina platensis. Indian J. Microbiol., 48, 3: 348352.

KHAN M., VARADHARA S., GANSESA, L.P., SHOBHA J.C., NAIDU, M.U. AND PARMANDI, N. L. 2005. "C-Phycocyanin protects against ischemia-reperfusion injury of the heart through the involvement of p38 and ERK signalling," Am. J. Physiol. Heart Circ. Physiol., 290(5): 2136-2145.

KUMAR V., BHATNAGAR A.K. AND SRIVASTAVA, J.N. 2011. Antibacterial activity of crude extracts of Spirulina platensis and its structural elucidation of bioactive compound. Journal of Medicinal Plants Research, 5(32): 7043-7048.

LEISTNER L. 2000. Basic aspects of food preservation by hurdle technology.Int. J. Food Microbiol.; 55(13):181-186.

MALA R., SAROJINI M., SARAVANABABU S. AND UMADEVI G. 2009. Screening for antimicrobial activity of crude extracts of Spirulina platensis. J Cell Tissue Res; 9 (3): 1951-1955.

McCARTY M.F. 2007. Clinical potential of Spirulina as a source of phycocyanobilin. J Med Food 10: 566-570.

McMEEKIN T.A., NICHOLS D.S., OLLEY J., GARDA H. AND BRENNER R.R. 2000. Effect of temperature and salinity stress on growth and lipid composition of Shewanella gelidimarina. Appl. Environ. Microbiol. ; 66(6):2422-2429.

MENDIOLA J.A., JAIME L. AND SANTOYO S. 2007. Screening of functional compounds in supercritical fluid extracts from Spirulina platensis. Food Chem. 102 (4): 1357-1367.

NAVACCHI M.F.P., MONTEIRO DE CARVALHO J. C., TAKEUCHI K P. AND DANESI E.D.G. 2012. Development of cassava cake enriched with its own bran and Spirulina platensis. Acta Scientiarum Technology (Maringá), 34 (4) 465- 472.

ÖZYURT G., LEYLA U., ILKNUR Y., SAADET G., GÖKÇE A., BURCU A. AND OYA I. 2015. Evaluation of the Cooking Quality Characteristics of Pasta Enriched with Spirulina Platensis. Journal of Food Quality, vol. 38 (4): 268-272

PRAKASH D R. AND KUMARI P. 2011. Preparation of low-fat and high-protein frozen yoghurt enriched with papaya pulp and Spirulina. Trends Biosci.4(2):182184.

PURNOMO H. AND RAHARDIYAN D. 2008. Review Article Indonesian Traditional Meatball. International Food Research Journal 15(2): 101-108

PYNE S.K., BHATTACHARJEE P. AND SRIVASTAV P. 2017. Microalgae (Spirulina Platensis) and Its Bioactive Molecules: Review. Indian Journal of Nutrition. 4(2): 1-6.

SAJILATA M., SINGHAL R. AND KAMAT M. 2007. A fraction of lipids and purification of -linolenic acid (GLA) from Spirulina Platensis. Food Chem., 109: 580-586.

SOHEILI M. AND KHhOSRAVI-DARANI K. 2011. The potential health benefits of algae and microalgae in medicine: A review on Spirulina platensis. Current Nutrition \& Food Science, 7 (4): 279-285.

USHARANI G, SARANRAJ P AND KANCHANA D. 2012. Spirulina Cultivation, A Review. International Journal of Pharmaceutical and Biological Archives, 3(6): 1327-1341.

USHARANI G., SRINIVASAN G., SIVASAKTHI S. AND SARANRAJ P. 2015. Antimicrobial activity of Spirulina platensis solvent extracts against 
pathogenic bacteria and fungi. Advan. Biol. Res., 9 (5): 292-298.

VELI G. AND YASEMIN B. 2012. Effect of olive leaf, blueberry and Zizyphus jujube extracts on the quality and shelf life of meatball during storage. Journal of Food, Agriculture \& Environment Vol.10 (2): 190195.

YILMAZ I. AND DEMIRCI M. 2010. Effect of Different Packaging Methods and Storage Temperature on Microbiological and Physicochemical Quality Characteristics of Meatball. Food Sci Tech Int.;16 (3):259-265.

XUE C., Hu Y., SAITO H., ZHANG Z., LI Z., CAI Y., OU C., LIN H. AND IMBS A.B. 2002. Molecular species composition of glycolipids from Spirulina platensis. Food Chemistry, 77 (1): 9-13.
How to cite this article:

Kareman S. Awadalla; Ali M. Ahmed;Mohamed M. Abdel-Daim; Nagwa T. Elshraway and Mariam A. Abdel-Wahab.2020. Evaluation of Sensory and Bacteriological Quality of Meatball Supplemented by Spirulina Platensis. Journal of Applied Veterinary Sciences, 5(2): 13-24. DOI:HTTPS://DX.DOI.ORG/10.21608/JAVS.2020.85554 